Supporting Information for

\title{
Biophysical Impact of Lipid A Modification Caused by Mobile Colistin Resistance Gene on Bacterial Outer Membranes
}

Wendong Ma ${ }^{1,2}$, Xukai Jiang 3 , Yujiang Dou ${ }^{1 *}$, Zhihong Zhang ${ }^{2}$, Jian Lit ${ }^{4}$, Bing Yuan ${ }^{5 *}$ and Kai Yang ${ }^{2 *}$

1 School of Electronic Information, Dongguan Polytechnic, Dongguan 523808, Guangdong, China

${ }^{2}$ Center for Soft Condensed Matter Physics and Interdisciplinary Research \& School of Physical Science and Technology, Soochow University, Suzhou 215006, Jiangsu,

China

${ }^{3}$ National Glycoengineering Research Center, Shandong University, Qingdao 266237, Shandong, China

${ }^{4}$ Biomedicine Discovery Institute, Infection Program, Department of Microbiology, Monash University, Melbourne, VIC 3800, Australia

${ }^{5}$ Songshan Lake Materials Laboratory, Dongguan 523808 Guangdong, China 


\section{Models and Methods}

Simulation system. CG models of 1-palmitoyl-2-oleoyl-sn-glycero-3phosphoethanolamine (POPE), 1-palmitoyl-2-oleoyl-sn-glycero-3-phosphorylglycerol (POPG), cardiolipin and lipid A were built based on the classical Martini force-field. ${ }^{1}$ The pEtN model was extracted from phosphatidylethanolamine (PE). Lipid A-pEtN was constructed by attaching one zwitterionic moiety, $\mathrm{pEtN}$, to one $\mathrm{PO}_{4}$ group of the pristine lipid A molecule (Figure 1a). ${ }^{2,3}$ This modification changes the net charge of lipid A from -4 to -3 at physiological $\mathrm{PH}$.

Our previous quantitative membrane lipidomics results ${ }^{4}$ were employed here to determine the phospholipid composition of the OM of Acinetobacter baumannii used in the simulations (i.e., outer leaflet: 75\% lipid A + lipid A-pEtN, 14.5\% POPE, 6.5\% POPG, and 4\% cardiolipin; inner leaflet: 58\% POPE, 26\% POPG, and 16\% cardiolipin; the number of different lipids in the OM under different conditions are shown in Table S1). In different simulations, the proportion of lipid A and lipid A-pEtN molecules, which together comprised $75 \%$ of the outer leaflet phospholipids, were varied from $100 \%$ lipid $\mathrm{A}$ and $0 \%$ lipid $\mathrm{A}-\mathrm{pEtN}$ (described in the main text as $0 \%$ lipid A-pEtN) to 25\% lipid A and 75\% lipid A-pEtN (described as 75\% lipid A-pEtN). Asymmetric OM models were developed using CHARMM-GUI. ${ }^{5}$ The size of the OM was $\sim 30 \mathrm{~nm} \times 30 \mathrm{~nm}$; this is sufficient for visualization of morphological changes. Membrane charges were neutralized using $0.1 \mathrm{M} \mathrm{CaCl}_{2}$ and additional $\mathrm{Ca}^{2+}$ ions were included in the system.

Simulation protocols. Simulations were performed using the GROMACS 2019.06 
package (www.gromacs.org) with CG MARTINI force field. ${ }^{1,6}$ All simulations were conducted in the semi-isotropic ensemble at constant temperature $(313 \mathrm{~K})$ and pressure (1.0 bar). The temperature was controlled using the velocity-rescaling thermostat with a time constant of $\tau=1.0 \mathrm{ps}$ and the pressure manipulated using a Parrinello-Rahman semi-isotropic barostat with a time constant of 12 ps and compressibility of $4.5 \times 10^{-5}$ $\operatorname{bar}^{-1} .{ }^{7,8}$ Periodic boundary conditions were applied in all three directions. At the beginning of each simulation, the bilayer membrane had a laterally random distribution of different components; a 150-ns equilibrium was performed for each system to achieve an equilibrate membrane state (Figure S1). All simulations were run for at least $2.5 \mu$ s with a time step of 20 fs. A minimum of three independent simulations were conducted for each system. 
Table S1. The total number of different lipids in the outer membrane (inner and outer leaflets combined) with different proportions (0-75\%) of pEtN-modified lipid A (i.e., lipid A-pEtN) in the outer leaflet.

\begin{tabular}{|c|c|c|c|c|c|}
\hline $\begin{array}{r}\text { Lipid } \\
\text { No of } \\
\text { lipid A-pEtN }\end{array}$ & Lipid A & $\begin{array}{c}\text { Lipid } \\
\text { A-pEtN }\end{array}$ & POPE & POPG & CDL2 \\
\hline 0 & 525 & 0 & 852 & 380 & 236 \\
\hline 10 & 473 & 52 & 852 & 380 & 236 \\
\hline 30 & 368 & 157 & 852 & 380 & 236 \\
\hline 50 & 263 & 262 & 852 & 380 & 236 \\
\hline 75 & 132 & 393 & 852 & 380 & 236 \\
\hline
\end{tabular}

pEtN, phosphoethanolamine; POPE, 1-palmitoyl-2-oleoyl-sn-glycero-3phosphoethanolamine; POPG, 1-palmitoyl-2-oleoyl-sn-glycero-3-phosphorylglycerol; CDL2, cardiolipin (16:0, 18:1-16:0, 18:1). In the simulations, the proportion of lipids in the outer membrane outer leaflet was 75\% lipid A + lipid A-pEtN, 14.5\% POPE, 6.5\% POPG, and 4\% cardiolipin; the proportion of lipids in the inner leaflet was 58\% POPE, $26 \%$ POPG, and $16 \%$ cardiolipin. 

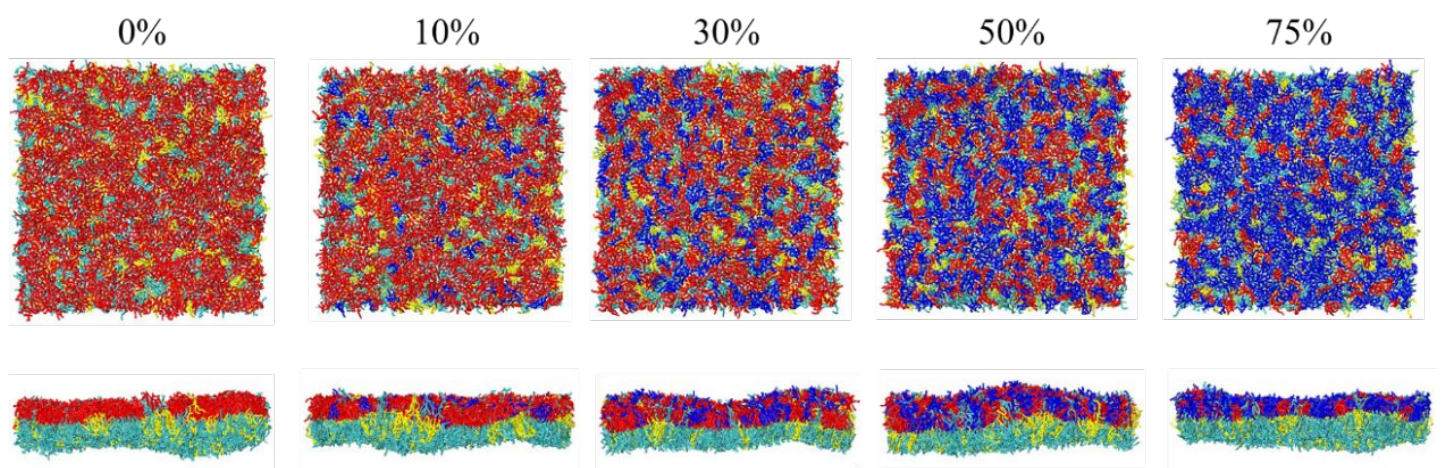

Figure S1. Initial states of the outer membrane (obtained from $t=1 \mathrm{~ns}$ ) with different proportions (0-75\%) of pEtN-modified lipid A (i.e., lipid A-pEtN) in the outer leaflet. Top: top view; Bottom: side view. Red, lipid A; Blue, lipid A-pEtN; Cyan, POPE and POPG; Yellow, cardiolipin.
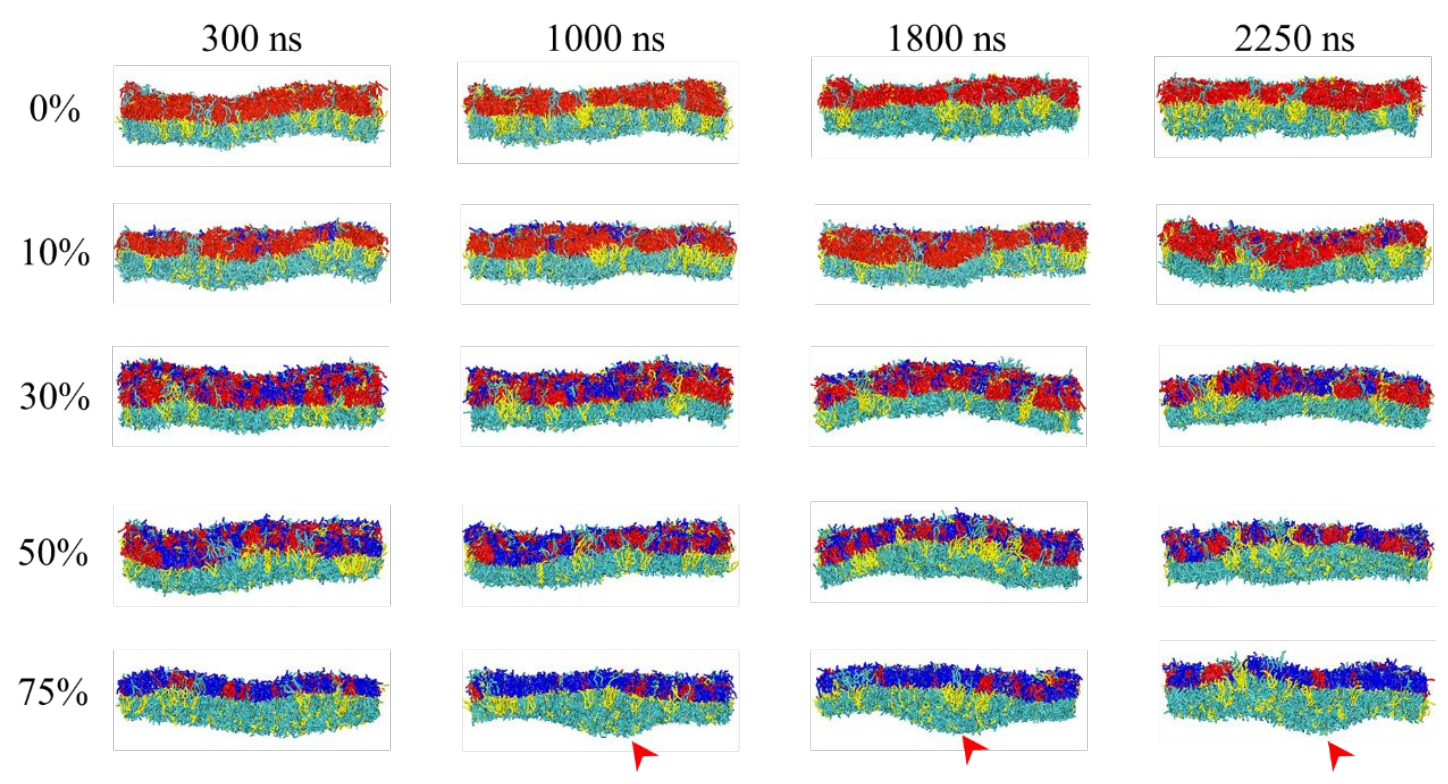

Figure S2. Snapshots of outer membrane states at various times up to 2,250 ns with different proportions $(0,10,30,50$ and $75 \%)$ of lipid A-pEtN in the outer leaflet. The negative curvature region with $75 \%$ lipid A-pEtN is indicated with red arrowheads. Red, lipid A; Blue, lipid A-pEtN; Cyan, POPE and POPG; Yellow, cardiolipin. 
$\mathbf{a}$

\section{Replica 1}
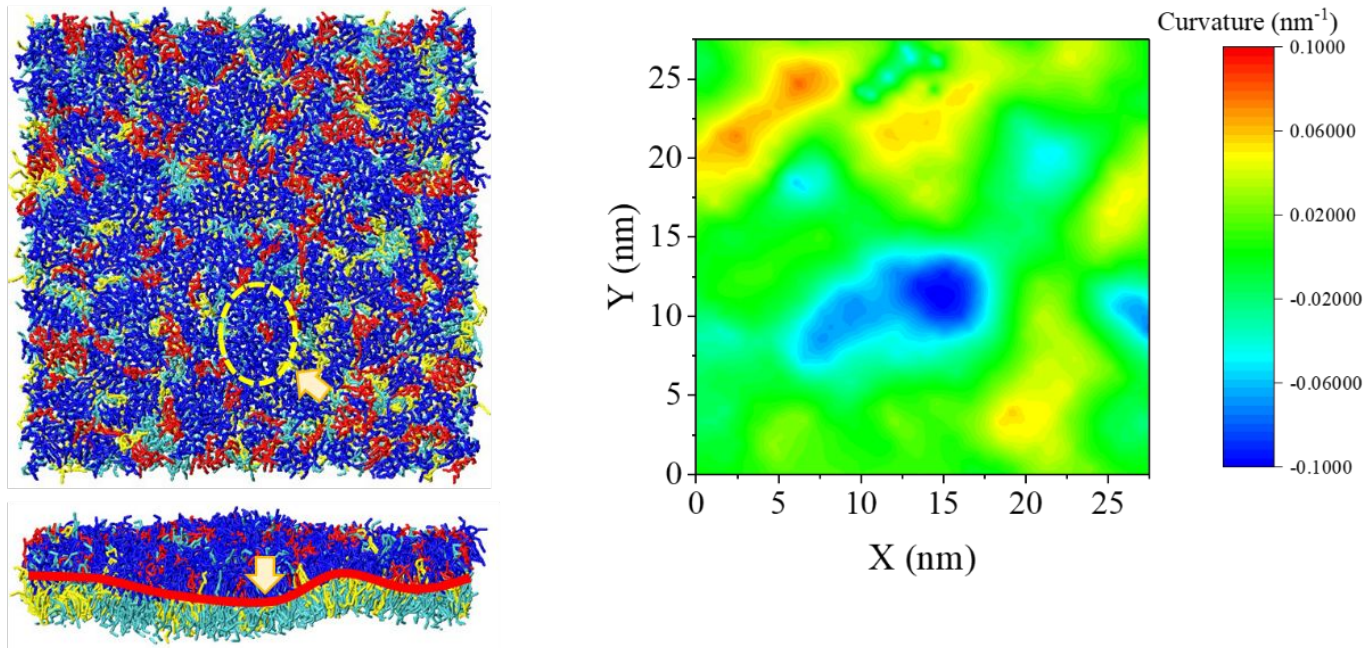

b

Replica 2
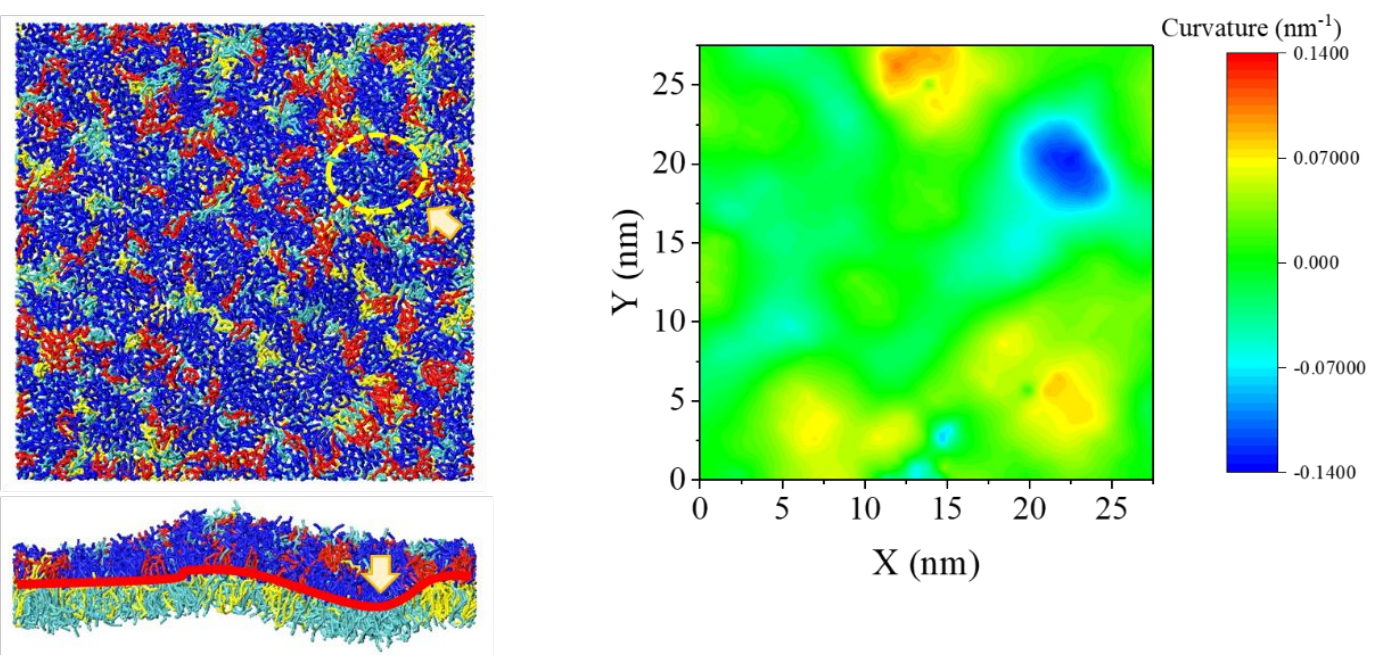

Figure S3. Two replicate simulations performed with 75\% lipid A-pEtN in the outer leaflet of the outer membrane. Spontaneously-formed negative membrane curvature (shown in yellow circles and arrows) was observed in both independent simulations. The enrichment of lipid A-pEtN and depletion of cardiolipin can be observed in these regions. Red, lipid A; Blue, lipid A-pEtN; Cyan, POPE and POPG; Yellow, cardiolipin. 


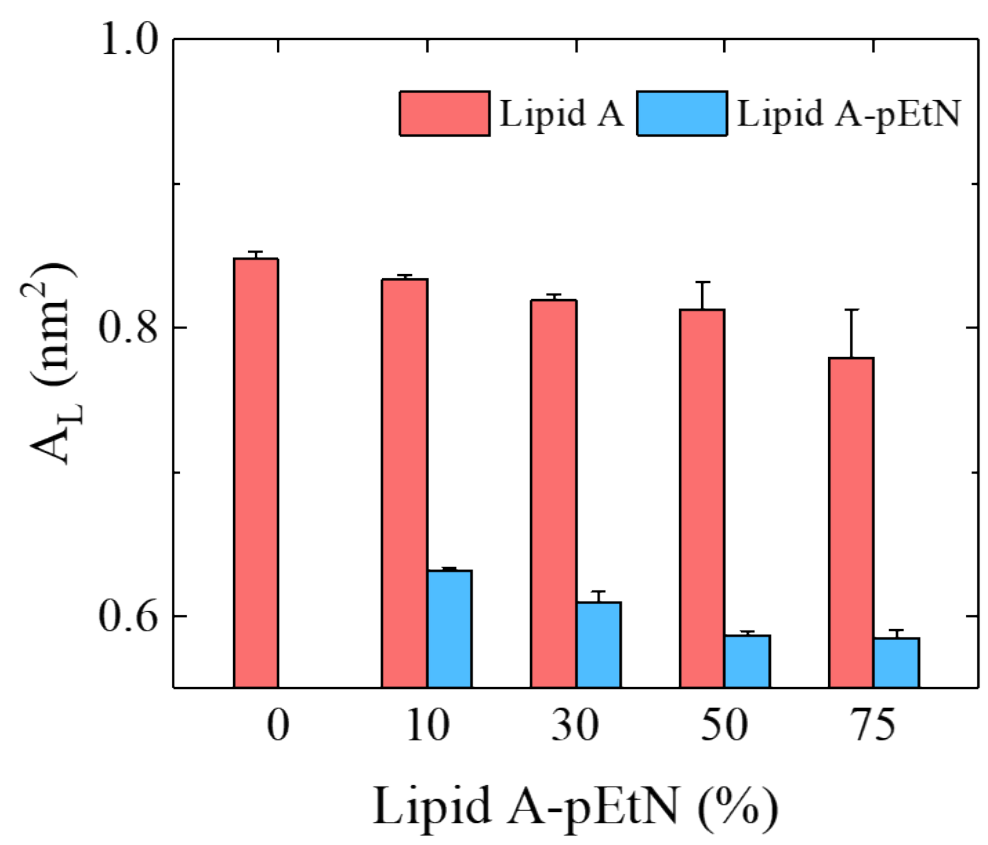

Figure S4. Mean area per lipid $\left(\mathrm{A}_{\mathrm{L}}\right)$ of lipid $\mathrm{A}$ and lipid A-pEtN with different proportions $(0,10,30,50$ and $75 \%)$ of lipid A-pEtN in the outer leaflet of the outer membrane. 
Lipid A
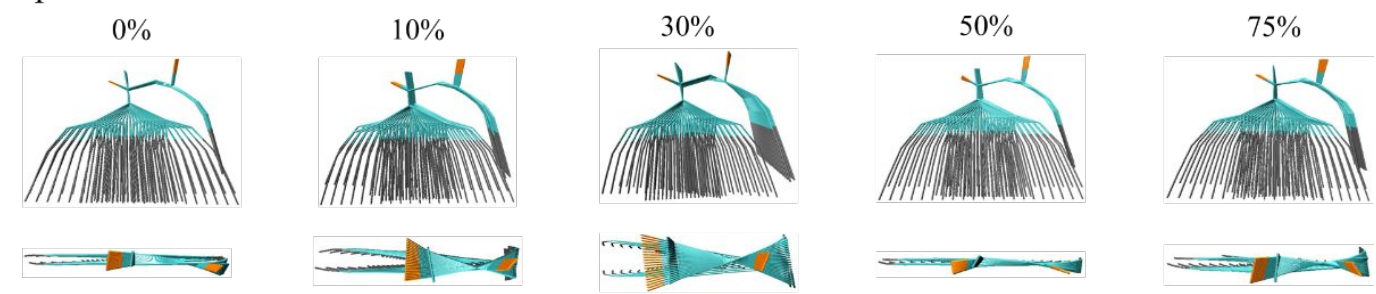

b

Lipid A-pEtN
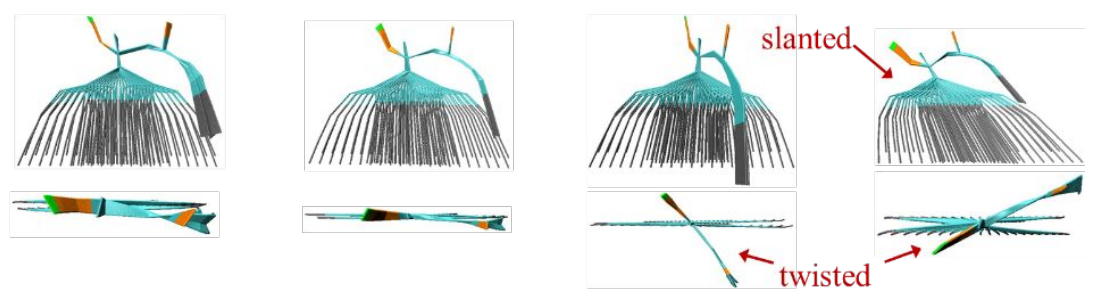

Figure S5. First principal component analysis illustrating the conformational changes of lipid A (a) and lipid A-pEtN (b) with different proportions (0, 10, 30, 50 and 75\%) of lipid A-pEtN in the outer leaflet of the outer membrane. Note that 20 molecules in each snapshot are shown to illustrate such changes. Top, side view; Bottom, top view. Cyan, head groups; Grey, tail groups; Orange, phosphate; Green, ethanolamine. 
a

Lipid A

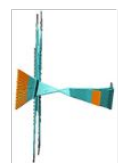

b

Lipid A-pEtN
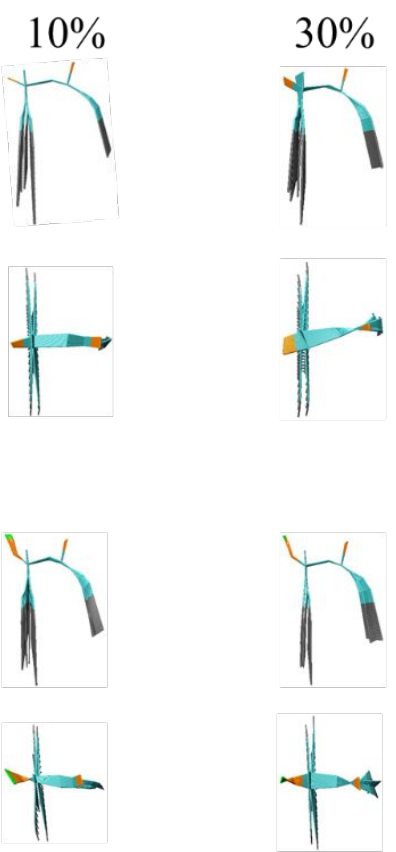
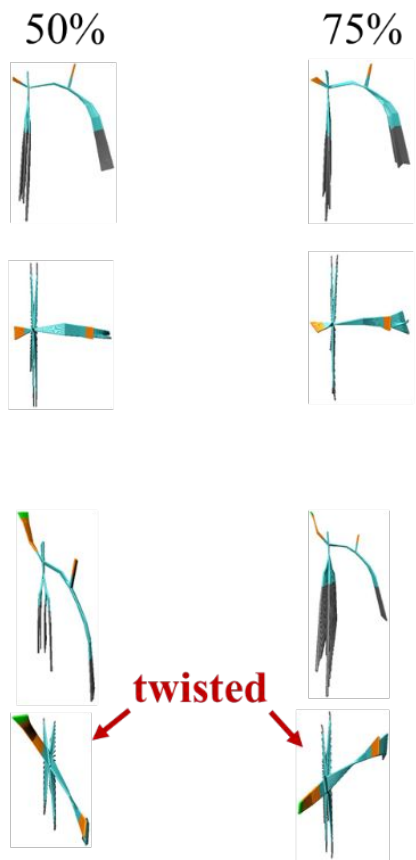

Figure S6. Second principal component analysis illustrating the conformational changes of lipid A (a) and lipid A-pEtN (b) with different proportions (0, 10, 30, 50 and $75 \%$ ) of lipid A-pEtN in the outer leaflet of the outer membrane. Note that 20 molecules in each snapshot are shown to illustrate such changes. Top, side view; Bottom, top view. Cyan, head groups; Grey, tail groups; Orange, phosphate; Green, ethanolamine. 


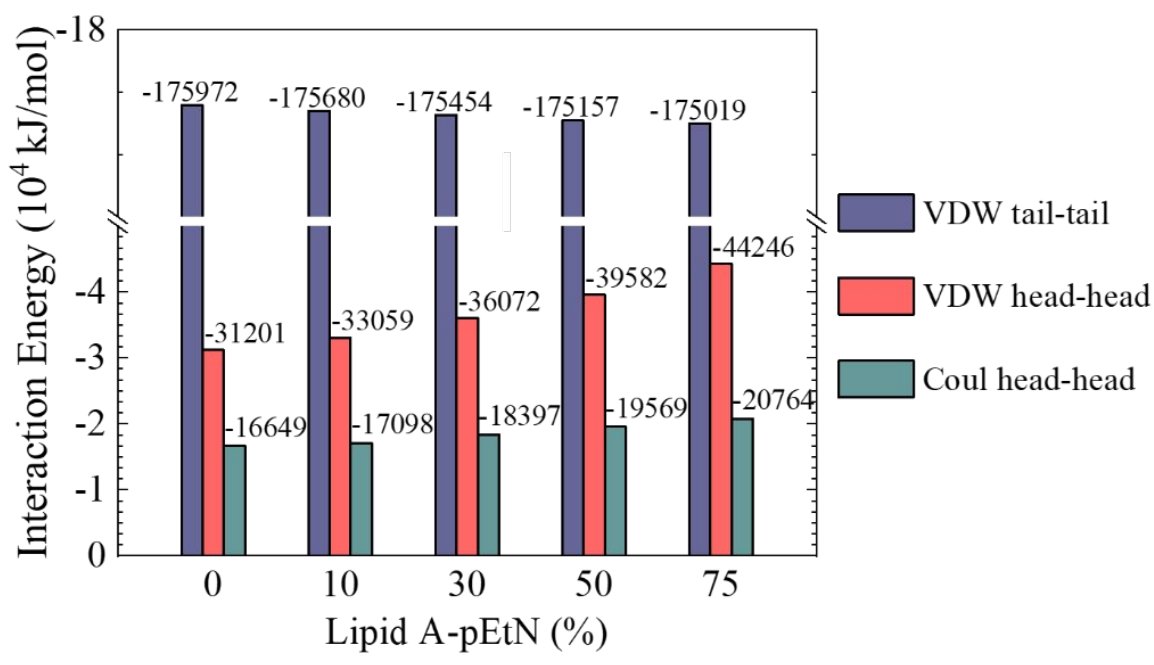

Figure S7. Histograms showing interaction energy of headgroups or tail groups of lipid $\mathrm{A}(-\mathrm{pEtN})$ with different proportions of lipid A-pEtN.

\section{Note:}

Calculation of the interaction energy between lipids in the outer leaflet revealed only a small increase $(\sim 0.5 \%)$ in interaction energy between the lipid tails as the proportion of lipid A-pEtN increased (Figure S7). In contrast, the interaction energy between lipid headgroups decreased by $\sim 42 \%$ as the proportion of lipid A-pEtN increased from 0 to $75 \%$ (Figure S7). This comparison shows that it is the headgroup modification of lipid A-pEtN that adjusts the packing state of lipids in the membrane (including $A_{L}$ ). 


\section{References}

(1) Marrink, S. J.; Risselada, H. J.; Yefimov, S.; Tieleman, D. P.; de Vries, A. H. The MARTINI Force Field: Coarse Grained Model for Biomolecular Simulations. $J$. Phys. Chem. B 2007, 111, 7812-7824.

(2) Jeannot, K.; Bolard, A.; Plésiat, P. Resistance to Polymyxins in Gram-Negative Organisms. Int. J. Antimicrob. Ag. 2017, 49, 526-535.

(3) Yang, Q.; Li, M.; Spiller, O. B.; Andrey, D. O.; Hinchliffe, P.; Li, H.; MacLean, C.; Niumsup, P.; Powell, L.; Pritchard, M.; Papkou, A.; Shen, Y.; Portal, E.; Sands, K.; Spencer, J.; Tansawai, U.; Thomas, D.; Wang, S.; Wang, Y.; Shen, J.; Walsh, T. Balancing Mcr-1 Expression and Bacterial Survival Is a Delicate Equilibrium between Essential Cellular Defence Mechanisms. Nat. Commun. 2017, 8, 2054.

(4) Zhu, Y.; Lu, J.; Han, M.; Jiang, X.; Azad, M. A. K.; Patil, N. A.; Lin, Y.; Zhao, J.; Hu, Y.; Yu, H. H.; Chen, K.; Boyce, J. D.; Dunstan, R. A.; Lithgow, T.; Barlow, C. K.; Li, W.; Schneider-Futschik, E. K.; Wang, J.; Gong, B.; Sommer, B.; Creek, D. J.; Fu, J.; Wang, L.; Schreiber, F.; Velkov, T.; Li, J. Polymyxins Bind to the Cell Surface of Unculturable Acinetobacter baumannii and Cause Unique Dependent Resistance. Adv. Sci. 2020, 7, 2000704.

(5) Lee, J.; Patel, D. S.; Ståhle, J.; Park, S.-J.; Kern, N. R.; Kim, S.; Lee, J.; Cheng, X.; Valvano, M. A.; Holst, O.; Knirel, Y. A.; Qi, Y.; Jo, S.; Klauda, J. B.; Widmalm, G.; Im, W. CHARMM-GUI Membrane Builder for Complex Biological Membrane Simulations with Glycolipids and Lipoglycans. J. Chem. Theory Comput. 2019, 15, 775-786.

(6) Abraham, M. J.; Murtola, T.; Schulz, R.; Pall, S.; Smith, J. C.; Hess, B.; Lindahl, E. GROMACS: High Performance Molecular Simulations through Multi-Level Parallelism from Laptops to Supercomputers. SoftwareX 2015, 1-2, 19-25.

(7) Bussi, G.; Donadio, D.; Parrinello, M. Canonical Sampling through Velocity Rescaling. J. Chem. Phys. 2007, 126, 014101.

(8) Parrinello, M.; Rahman, A. Polymorphic Transitions in Single Crystals: A New Molecular Dynamics Method. J. Appl. Phys. 1981, 52, 7182-7190. 